\title{
SHOT NOISE BEHAVIOUR OF SUBTHRESHOLD MOS TRANSISTORS
}

\author{
J. FELLRATH \\ CEH, rue Breguet 2, Neuchâtel, Switzerland
}

Résumé. - On montre, qu'en faible inversion, l'asymptote petits courants de la résistance équivalente de bruit ramenée à la grille vaut $\frac{n^{2}}{2} \frac{U_{\mathrm{T}}}{I_{\mathrm{D}}}$ et qu'elle correspond à un bruit de grenaille. Des mesures confirmant cette théorie ainsi que des mesures de bruit de scintillement sont présentées. On utilise des transistors à canaux $\mathbf{p}$ et $\mathbf{n}$ intégrés en technologie CMOS à grille de silicium sur silicium massif et sur saphir.

\begin{abstract}
It is shown that assuming weak inversion, low drain current asymptotic value of the gate equivalent noise resistor is given by $\frac{n^{2}}{2} \frac{U_{\mathrm{T}}}{I_{\mathrm{D}}}$, corresponding to shot noise. Measurements confirming this theory as well as flicker noise measurements on $\mathrm{n}$ and $\mathrm{p}$ channel transistors integrated with either bulk or SOS CMOS silicon gate technology are presented.
\end{abstract}

1. Introduction. - The known weak-inversion behaviour [1, 2] of the MOS transistor assumes that the drain current is solely due to diffusion of the carriers. The related shot noise can be easily evaluated. In addition to shot noise, it is known that MOS transistors exhibit flicker noise [3, 4]. Flicker noise is negligible if the transistor is operated at very low drain current.

Measurements on both $\mathrm{n}$ and $\mathrm{p}$ devices realized with various technologies confirm the theoretical results.

2. Noise model. - In order to find a noise model containing independent noise sources, we must divide the d.c. currents into physically distinct components and then identify a separate noise generator for each distinguishable group of carriers.

Troutman and Chakravarti [2] have shown that diffusion dominates channel current in MOS transistors in weak inversion. The diffusion current injected by the source-to-substrate junction flows near the surface with a very long diffusion length and is collected by the drain. Neglecting the generation currents in the space charge region of source, channel and drain, the current remains as only component.

The shot noise current related to the diffusion of carriers is given by

$$
i_{\mathrm{DN}}^{2}=2 q I_{\mathrm{D}} \Delta f
$$

where $q$ is the charge of electron, $\Delta f$ the bandwidth and $I_{\mathrm{D}}$ the drain current which can, according to its physical origin, be represented by a current source $i_{\mathrm{DN}}^{2}$ between source and drain. It gives rise to a white noise spectrum.

When the gate to source voltage of a MOS transistor is reduced below the extrapolated threshold value, the transistor enters the weak inversion region. According to [1] the drain current is given by :

$$
\begin{aligned}
I_{\mathrm{D}}=S I_{\mathrm{DO}} & \exp \left(\frac{V_{\mathrm{G}}}{n U_{\mathrm{T}}}\right) \\
& \times\left(\exp -\left[\frac{V_{\mathrm{S}}}{U_{\mathrm{T}}}\right]-\exp -\left[\frac{V_{\mathrm{D}}}{U_{\mathrm{T}}}\right]\right)
\end{aligned}
$$

where $V_{\mathrm{G}}, V_{\mathrm{S}}$ and $V_{\mathrm{D}}$ are the gate, source and drain voltages with respect to substrate; $S$ is a shape factor, $I_{\text {DO }}$ a characteristic current, $n$ a slope factor and $U_{\mathrm{T}}=k T / q$ ( $k$ Boltzman is constant, $T$ temperature).

For transistors operating in saturation, i.e. $V_{\mathrm{D}} \gg U_{\mathrm{T}}$ one defines a gate and a source transconductance

$$
\begin{gathered}
g_{\mathrm{mG}}=\frac{\partial I_{\mathrm{D}}}{\partial V_{\mathrm{G}}}=\frac{I_{\mathrm{D}}}{n U_{\mathrm{T}}} \quad \text { gate transconductance } \\
g_{\mathrm{mS}}=\frac{\partial I_{\mathrm{D}}}{\partial V_{\mathrm{S}}}=\frac{I_{\mathrm{D}}}{U_{\mathrm{T}}} \quad \text { source transconductance } .
\end{gathered}
$$

A convenient way of expressing noise is an equivalent input noise resistance $R_{\mathrm{N}}$, which is related to the input noise voltage through the relation

$$
U_{\mathrm{N}}^{2}=4 k T \Delta f R_{\mathrm{N}}
$$

$R_{\mathrm{N}}$ can easily be measured.

The drain noise current $i_{\mathrm{DN}}^{2}$ can then be referred to either the gate or the source. For transistors operating in saturation, i.e. $V_{\mathrm{D}} \gg U_{\mathrm{T}}$, the equivalent input shot noise resistances, referred to the gate or the source, are

$$
R_{\mathrm{NG}}=\frac{2 q I_{\mathrm{D}} \Delta f}{4 k T \Delta f} \frac{1}{g_{\mathrm{mG}}^{2}}=\frac{n}{2} \frac{1}{g_{\mathrm{mG}}}=\frac{n^{2}}{2} \frac{U_{\mathrm{T}}}{I_{\mathrm{D}}}
$$

and

$$
R_{\mathrm{NS}}=\frac{2 q I_{\mathrm{D}} \Delta f}{4 k T \Delta f} \frac{1}{g_{\mathrm{mS}}^{2}}=\frac{1}{2} \frac{1}{g_{\mathrm{mS}}}=\frac{1}{2} \frac{U_{\mathrm{T}}}{I_{\mathrm{D}}}
$$

respectively, where $g_{\mathrm{mG}}, g_{\mathrm{ms}}$ and $n$ are defined above. The MOS transistor exhibits an other noise component, the flicker $(1 / f)$ noise, caused by random trapping of free carriers in surface states, which modulates the channel conductivity and hence produces fluctuations in drain current $[3,4]$.

Flicker $(1 / f)$ noise, which also exists in strong inversion, can similarly be represented by an equivalent 
input noise resistor. From references [3] and [4], we obtain the equivalent input flicker noise resistance referred to the gate

$$
R_{\mathrm{FG}} \propto \frac{1}{f} \cdot \frac{1}{W L} \quad(W L=\text { gate area })
$$

if we suppose that the flicker noise is of the same nature in the strong or weak inversion.

The total equivalent input noise resistance is the sum of $R_{\mathrm{N}}$ and $R_{\mathrm{F}}$.

$R_{\mathrm{NG}}$ decreases with drain current whereas $R_{\mathrm{FG}}$ is constant : it is thus always possible by varying the drain current or the measuring frequency to distinguish the two noise components. For low currents or high frequency, shot noise is dominant while flicker noise dominates at high current or low frequency.

3. Measurement system. - The purpose of our work is to confirm experimentally this model. In order to neglect flicker noise, one has to work at very small drain currents. Stray capacitances have to be cancelled carefully. A low noise negative feedback amplifier was designed for this purpose.

Apart this amplifier, the measurement system is conventional and given in figure 1. Accurate measurements are possible for drain currents from $1 \mathrm{nA}$ to $3 \mathrm{~mA}$ and frequencies from $300 \mathrm{~Hz}$ to $100 \mathrm{kHz}$.

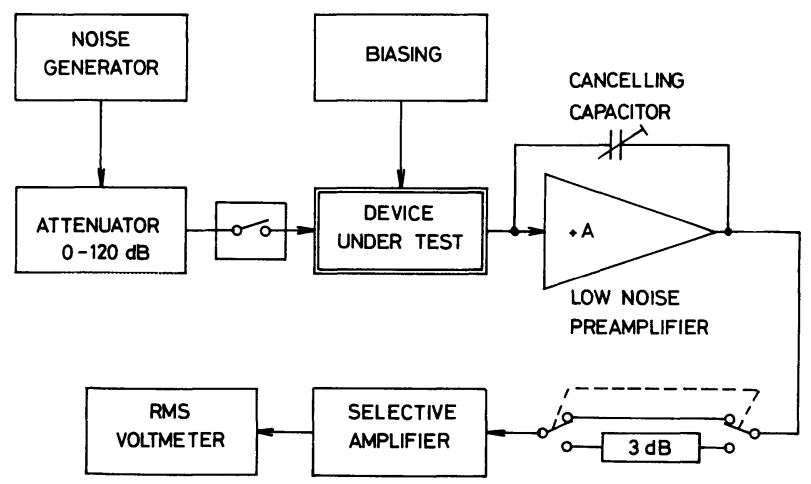

FIG. 1. - Schematic diagram of the measurement system used for the determination of the equivalent noise resistance.

4. Physical and electrical device parameters. 4.1 CMOS SILICON GATE BULK TECHNOLOGY (similar to [5]).

Substrate : Si orientation $\langle 100\rangle$

$$
N_{\text {D }}=(2 \pm 1) 10^{15} / \mathrm{cm}^{3} \text {. }
$$

P-Well : $N_{\mathrm{A}}$ (surface) $(4 \pm 1) 10^{15} / \mathrm{cm}^{3}$.

Gate oxide thickness : $75 \pm 6 \mathrm{~nm}$.

Strong inversion :

n-ch p-ch

MOS gain factor

$W / L=12 / 6 \mu \mathrm{m} \quad \beta \quad 85 \quad 30 \mu \mathrm{A} / \mathrm{V}^{2}$

Weak inversion :

Slope factor
4.2 CMOS SILICON GATE SOS TECHNOLOGY. $\mathrm{Si}$ epitaxial layer, orientation $\langle 100\rangle$ thickness $(0.6 \pm 0.1) \mu \mathrm{m}$

$$
\begin{aligned}
& N_{\mathrm{A}} \text { (surface) }(1 \pm 0.5) 10^{16} / \mathrm{cm}^{3}(\mathrm{n}-\mathrm{ch}) \\
& N_{\mathrm{D}} \text { (surface) }(1.2 \pm 0.6) 10^{15} / \mathrm{cm}^{3}(\mathrm{p}-\mathrm{ch})
\end{aligned}
$$

Gate oxide thickness : $75 \pm 6 \mathrm{~nm}$.

Strong inversion :

MOS gain factor

$$
W / L=12 / 5 \mu \mathrm{m} \quad \beta \quad 60 \quad 40 \mu \mathrm{A} / \mathrm{V}^{2}
$$

Weak inversion :

Slope factor

n 2

5. Experimental data on MOS transistors. - Experimental results are presented for various transistors realized with a CMOS bulk and SOS silicon gate technology.

The variation of the input noise resistance with drain current and frequency is given. The transistors are operated in saturation and the measuring frequency is chosen such that the parasitic polysilicon gate series resistance can be neglected.

A first essential conclusion can be drawn from the data shown in figures $2,3,5,6,8,9$ confirming our model.

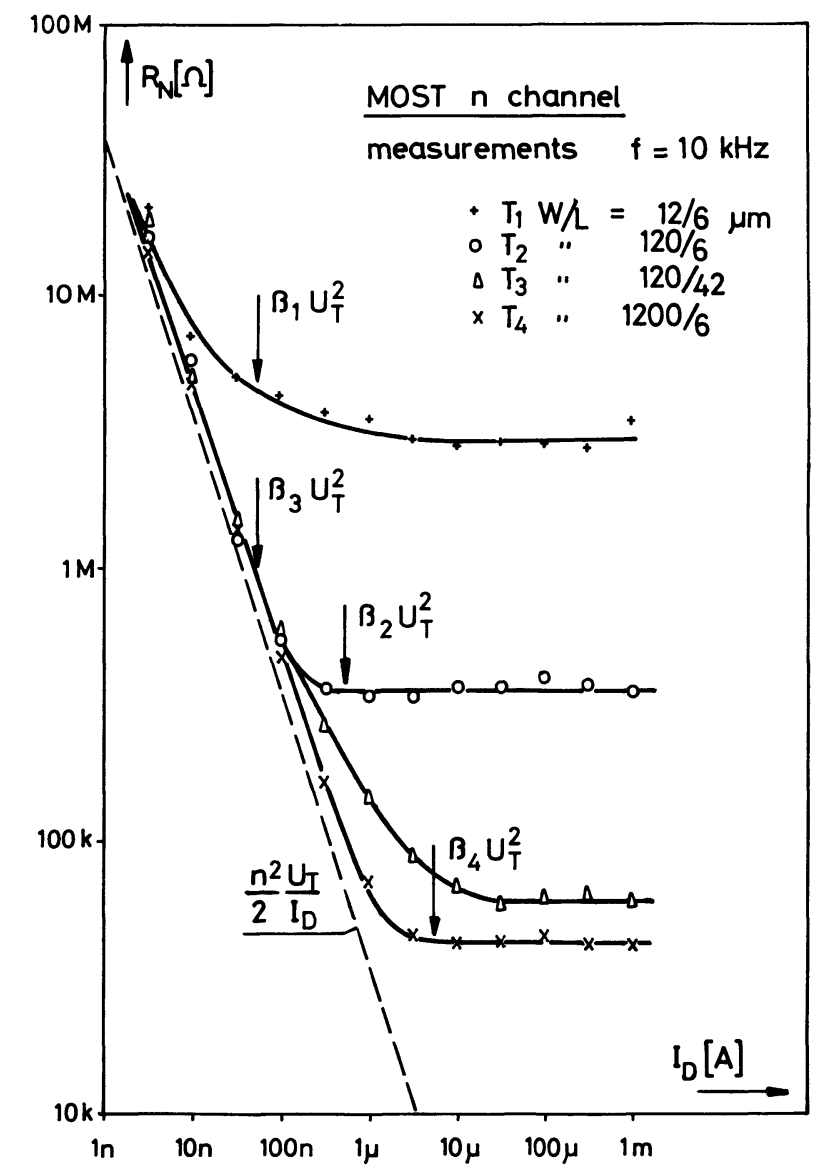

Fig. 2. - Noise equivalent gate resistance $R_{\mathrm{N}}$ versus drain current for Si bulk $n$ channel transistors of different shape factors. 


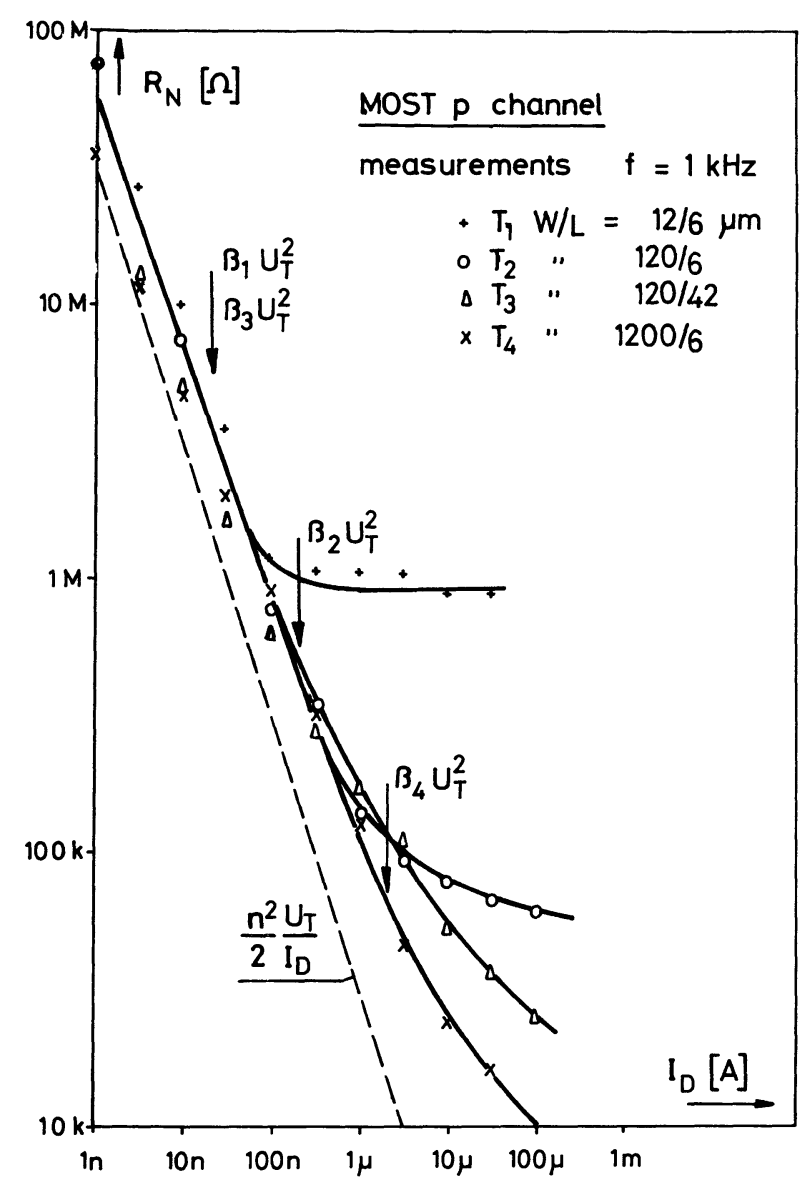

FIG. 3. - Noise equivalent gate resistance $R_{\mathrm{N}}$ versus drain current for Si bulk p channel transistors of different shape factors. Transistors of figures 2 and 3 provide from the same chip.

We notice that all the transistors have the predicted low current asymptote, given by equation (6), which corresponds to shot noise.

The same set of figures shows that the high current equivalent noise input resistance does not depend on current. The value decreases with the gate area $W L$ and depends upon the channel conductance and the technology.

We notice that, reduced to the same frequency and shape factor, the high current noise resistance (asymptotic value) representing flicker noise is roughly :

- for Si bulk technology one order of magnitude higher for $\mathrm{n}$ channel than for $\mathrm{p}$ channel,

- for SOS technology the same for $\mathrm{p}$ and $\mathrm{n}$ channel,

- three order of magnitude higher for SOS technology than for p channel bulk technology.

FIG. 4. - Noise equivalent gate resistance $R_{\mathrm{N}}$ versus frequency for $\mathrm{Si}$ bulk $\mathrm{p}$ and $\mathrm{n}$ channel transistors providing from the same chip.

FIG. 5. - Noise equivalent gate resistance $R_{\mathrm{N}}$ versus drain current for SOS $n$ channel transistors of different shape factors.
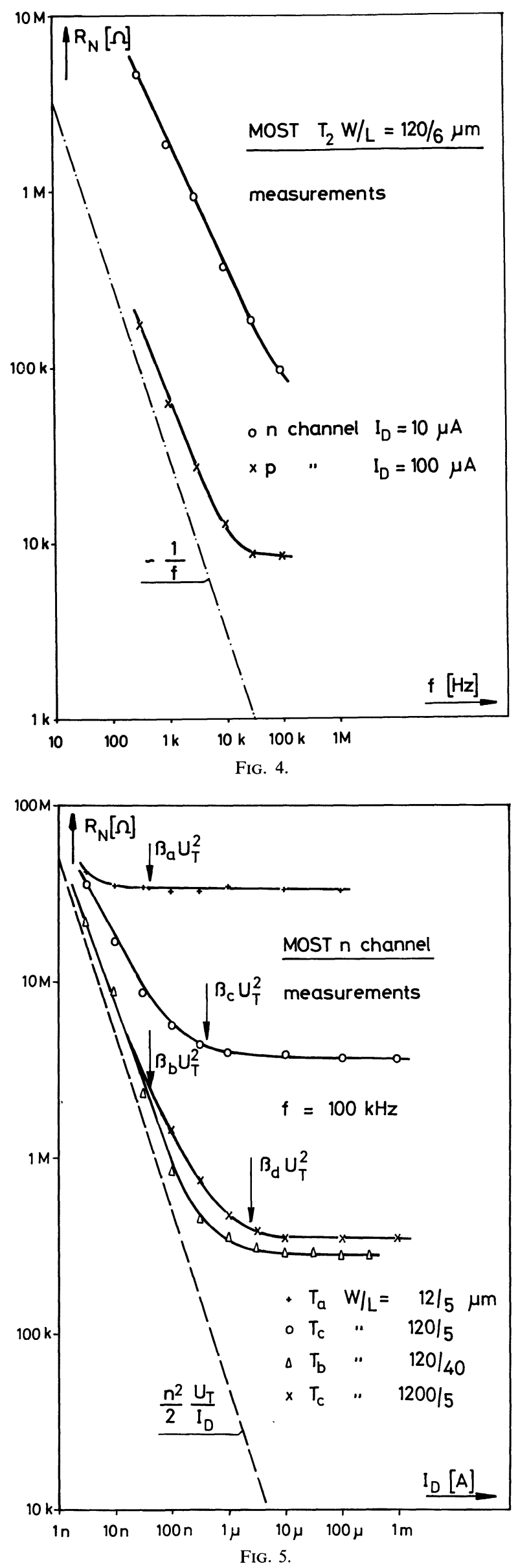

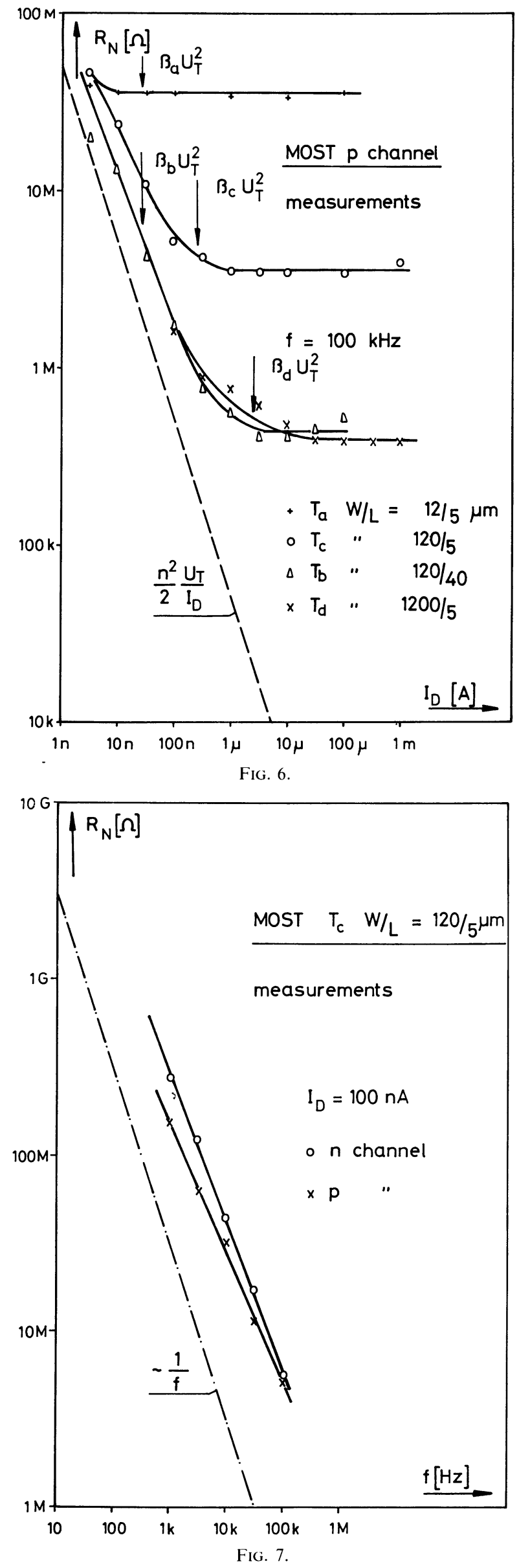

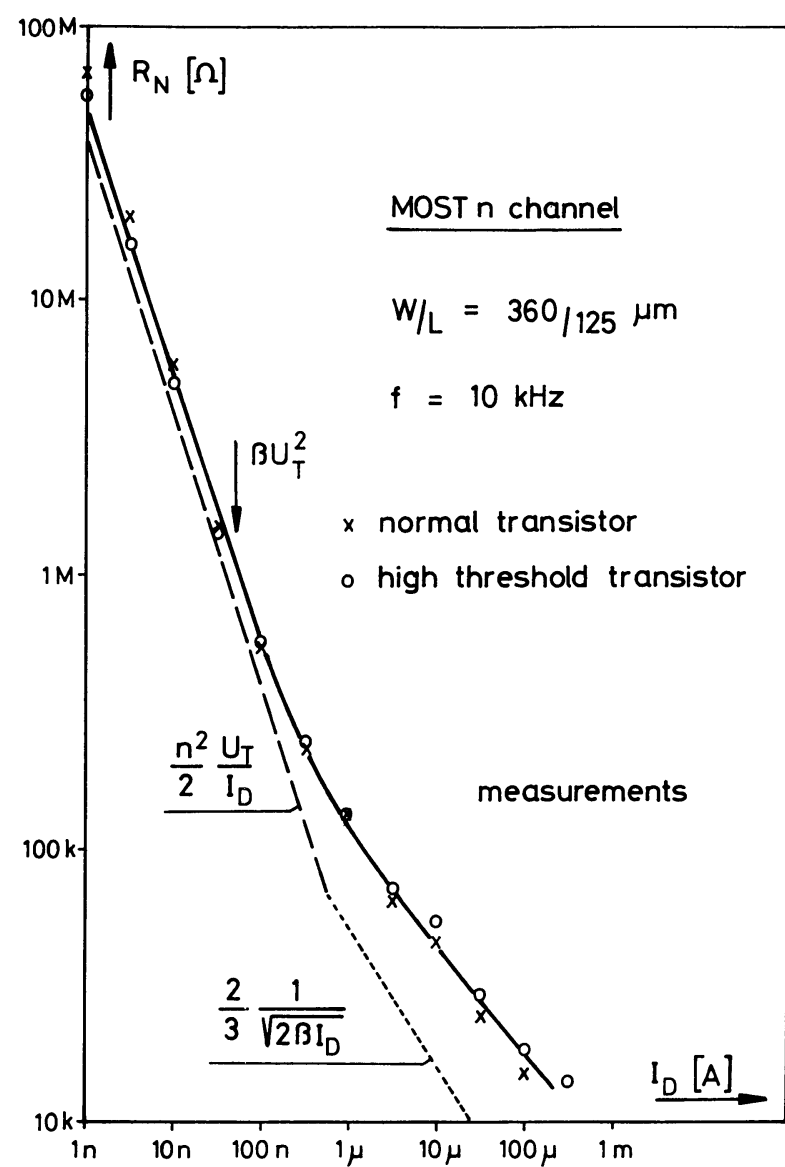

FIG. 8. - Noise equivalent gate resistance $R_{\mathrm{N}}$ versus drain current for Si bulk $\mathrm{n}$ transistors. Comparison between normal $\left(V_{\mathrm{T}}=0.35 \mathrm{~V}\right)$ and high threshold $\left(V_{\mathrm{T}}=1.7 \mathrm{~V}\right)$ transistors.

The measured data of figures 4,7 show an $1 / f$ dependence of the high current asymptote corresponding to flicker noise.

Figure 10 shows, in agreement with our model, that the equivalent source noise resistor is smaller than the equivalent gate resistance by a factor of $n^{2}$.

Figures 8 and 9 present measured data on transistors with $\mathrm{Si}$ bulk technology. These figures allow to compare normal transistors to high threshold transistors obtained by inverting the doping of the polysilicon gate.

The shot noise is the same for the four samples. The known [6] strong inversion asymptote

$$
R_{\mathrm{N}}=\frac{2}{3} \frac{1}{\sqrt{2 \beta I_{\mathrm{D}}}}
$$

FIG. 6. - Noise equivalent gate resistance $R_{\mathrm{N}}$ versus drain current for SOS p channel transistors of different shape factors. Transistors of figures 5 and 6 provide from the same chip.

FIG. 7. - Noise equivalent gate resistance $R_{\mathrm{N}}$ versus frequency for SOS $\mathrm{p}$ and $\mathrm{n}$ channel transistors providing from the same chip. 


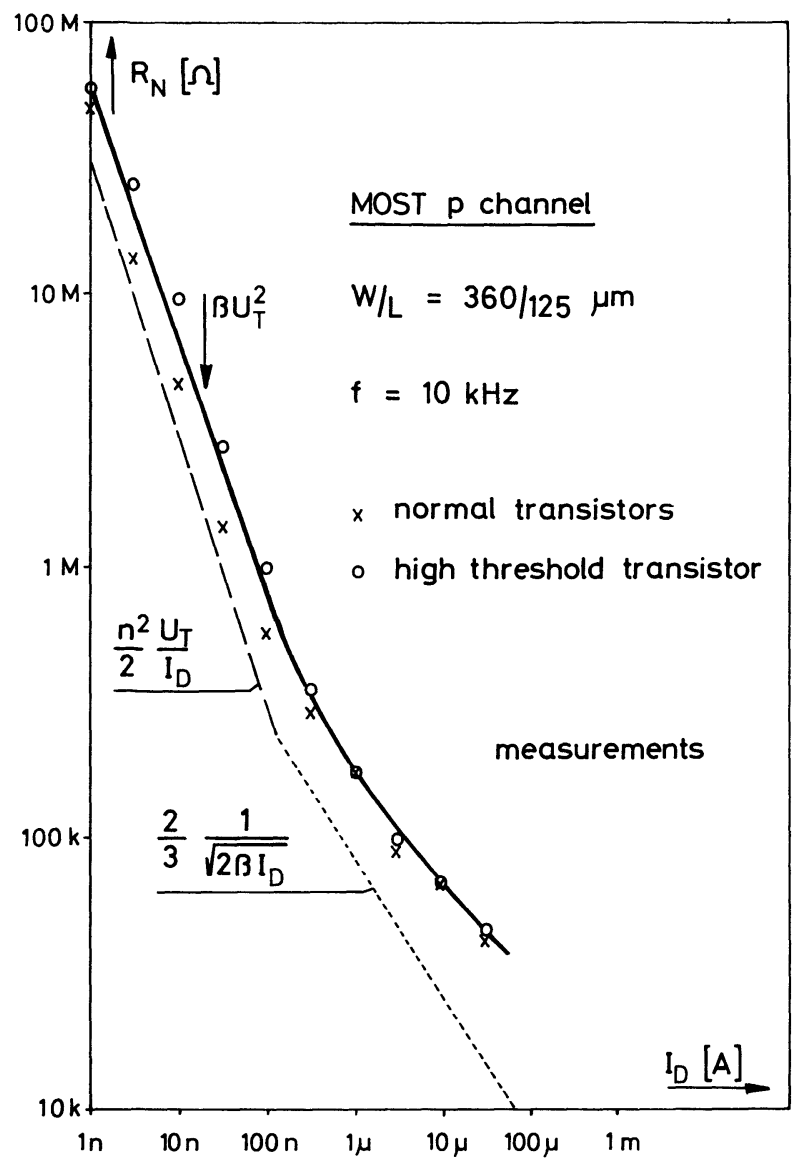

Fig. 9. - Noise equivalent gate resistance $R_{\mathrm{N}}$ versus drain current for Si bulk $\mathrm{p}$ transistors. Comparison between normal $\left(V_{\mathrm{T}}=0.25 \mathrm{~V}\right)$ and high threshold $\left(V_{\mathrm{T}}=1.8 \mathrm{~V}\right)$ transistors.

corresponding to thermal channel noise is also represented. A noticeable contribution of thermal noise can be seen on these figures.

6. Conclusion. - A very simple analysis can be made in order to characterize the shot noise behaviour of weak inversion MOS transistors leading to the interesting result that the low current asymptotic value of noise resistance depends only upon the exponential slope value. This result is confirmed by measurements on either bulk or SOS CMOS techno-

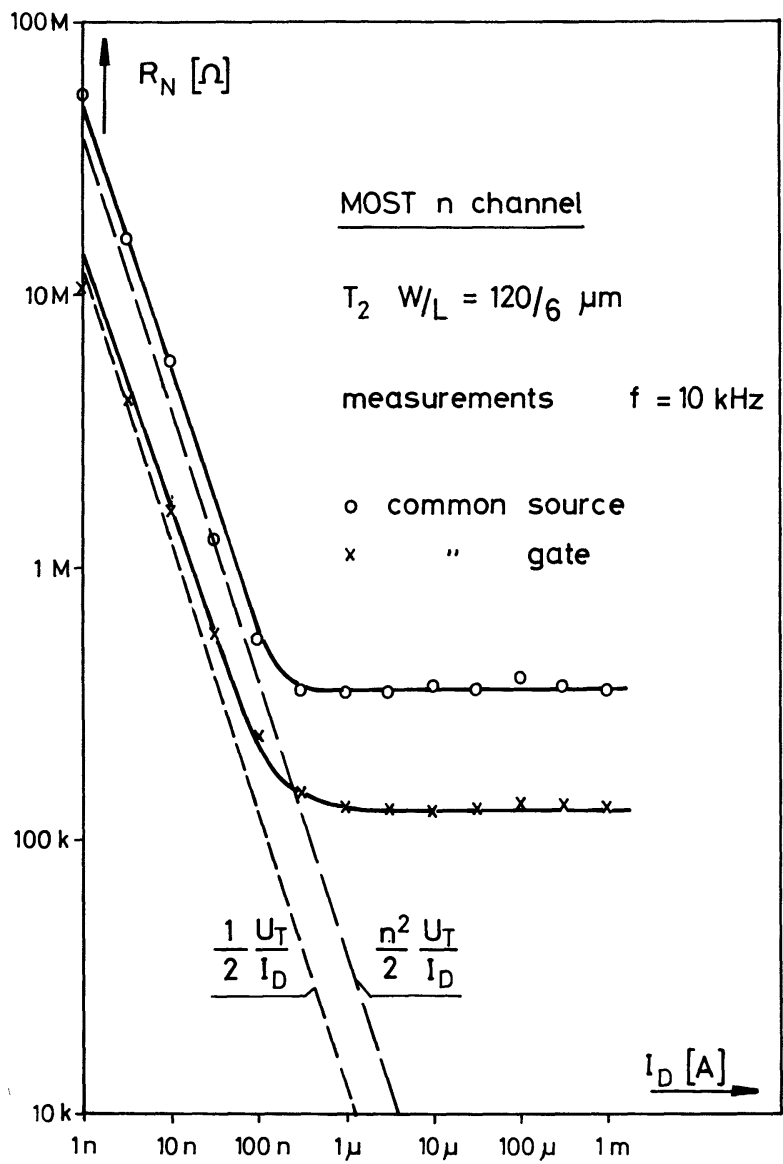

Fis. 10. - Noise equivalent gate resistance $R_{\mathrm{N}}$ versus drain current for Si bulk $\mathrm{n}$ channel transistors. Comparison between common source and common gate circuits.

logy. From a circuit designer point of view and without conditions on the value of capacitances, we notice that it is always possible to design a transistor, for which the flicker noise component is kept negligible. The remaining problem is then only the matching between the equivalent input noise resistance and the source impedance.

Acknowledgments. - The author wishes to thank Mr. G. Stähli for the valuable help supplied for the measurements.

\section{References}

[1] Vittoz, E., Fellrath, J., CMOS analog integrated circuits based on weak inversion operation, IEEE J. Solid State Circuits 12 (1977) 224-231.

[2] Troutman, R. R., Chakravarti, S. N., Subthrešshold characteristics of insulated-gate field-effect transistors, IEEE Trans. Circuits 12 (1977) 224-231.

[3] DAS, M. B., MOORE, J. M., Measurements and interpretation of low frequency noise in FET's, IEEE Trans. Electron Devices 21 (1974) 247-257.
[4] Hsu, S. T., Surface state related $1 / f$ noise in MOS transistors, Solid State Electron. 13 (1970) 1451-1459.

[5] Vittoz, E., Gerber, B. and Leuenberger, F., Silicongate CMOS frequency divider for the electronic wrist watch, IEEE J. Solid State Circuits 7 (1972) 100-104.

[6] Coвbold, R., Theory and application of FET (J. Wiley and Son London) 1970 , paragraph 9.3 , pp. $335-342$. 